Visible cathodoluminescence from mechanically milled germanium

This article has been downloaded from IOPscience. Please scroll down to see the full text article.

2002 Semicond. Sci. Technol. 171267

(http://iopscience.iop.org/0268-1242/17/12/310)

View the table of contents for this issue, or go to the journal homepage for more

Download details:

IP Address: 147.96.14.16

The article was downloaded on 21/02/2013 at 13:59

Please note that terms and conditions apply. 


\title{
Visible cathodoluminescence from mechanically milled germanium
}

\author{
E Nogales ${ }^{1}$, A Montone $^{2}$, F Cardellini ${ }^{2}$, B Méndez ${ }^{1}$ and \\ J Piqueras ${ }^{1}$ \\ ${ }^{1}$ Departamento de Física de Materiales, Facultad de Ciencias Físicas, Universidad \\ Complutense, E-28040 Madrid, Spain \\ ${ }^{2}$ ENEA-INN-NUMA, CR Casaccia, CP 2400, IT-00100 Roma, Italy
}

Received 9 September 2002, in final form 16 October 2002

Published 7 November 2002

Online at stacks.iop.org/SST/17/1267

\begin{abstract}
There has been interest in the past years in the visible luminescence properties of germanium nanocrystals. Most of the previous works refer to Ge nanocrystals embedded in an oxide matrix. In this work nanocrystalline germanium has been prepared by high-energy ball milling of Ge single crystals and Ge powder. The structure of the milled samples has been assessed by $\mathrm{x}$-ray diffraction (XRD) and transmission electron microscopy (TEM) while the luminescence has been investigated by cathodoluminescence (CL) in the scanning electron microscope (SEM). The samples consist of a powder of particles with sizes of hundreds of nanometres, normally aggregated to form larger particles. TEM reveals that the particles consist of nanocrystals with a wide range of sizes including crystallites of some nanometres. Milled Ge shows two CL bands at about 2.4-2.5 eV and $3.1 \mathrm{eV}$, respectively. Both emissions appear to be related to the presence of $\mathrm{Ge}$ nanocrystals. The $\mathrm{CL}$ of untreated and milled $\mathrm{GeO}_{2}$ powder has been also investigated for comparison.
\end{abstract}

\section{Introduction}

The influence of quantum confinement on the optical properties of nanocrystalline semiconductors is a subject of increasing interest due to the possibility of new applications to electronic devices. In particular, luminescence of nanocrystalline silicon (nc-Si) in the visible range has been extensively investigated. The most frequently referred form of nc-Si is porous silicon (for a review see [1]), but other systems, such as nc-Si formed by $\mathrm{Si}$ implantation in $\mathrm{SiO}_{2}$ [2] or nc-Si recrystallized from amorphous phase [3], also show visible luminescence. Many of the experimental results on the visible luminescence of nc-Si have been explained in the frame of a quantum confinement model in which the influence of the electronic states at the crystallites surface has to be considered, e.g., [4-6].

Germanium nanocrystals have also been found to show visible luminescence. Kanemitsu et al [7] reported visible photoluminescence of Ge nanocrystals in $\mathrm{SiO}_{2}$ glassy matrix and attributed it to a new structure with direct optical transition. Maeda [8] also investigated Ge nanocrystals in the same matrix and found his experimental photoluminescence (PL) data to be consistent with a quantum confinement model. Other authors have reported the appearance of PL emission related to the presence of Ge nanocrystals embedded in oxide matrix [9-14] or in colloidal form [15]. A number of results show the influence of defect states in the nanocrystals or in the interface nanocrystal-oxide on the observed photon emission, but the exact mechanism of luminescence of nc-Ge is under discussion. The existence of nanocrystalline $\mathrm{Si}$ and $\mathrm{Ge}$ related luminescence open new possibilities for group IV semiconductors as optoelectronic materials. In particular, one of the methods used to produce nanocrystals, mechanical ball milling, is potentially useful for large-scale applications of nanocrystalline Si and Ge. Mechanical milling has been frequently used to prepare nanocrystalline metallic materials [16] but it has not been often applied to the case of semiconductors [17-21]. Shen et al [20] reported PL in milled silicon and attributed the emission to silicon oxide phases at the surface region of the Si particles. In [21] the observed cathodoluminescence (CL) of milled silicon was explained by 
the presence of nanocrystals through a quantum confinement effect.

Whelan [17] reported the formation of Ge nanocrystals by ball milling and investigated the resulting structure by Raman and transmission electron microscopy (TEM) techniques. In the present work the structural and luminescence properties of ball-milled germanium have been investigated by XRD, TEM and $\mathrm{CL}$ in the scanning electron microscope.

\section{Experimental method}

A p-type single crystalline $\mathrm{Ge}$ wafer of electronic grade purity as well as Ge powder with a purity of $99.999 \%$ were used as starting material for the milling treatments. In order to study the possible influence of germanium oxide on the luminescence measurements, $\mathrm{GeO}_{2}$ powder was also milled under the same experimental conditions. Milling was performed at room temperature in an air cooled, high-energy Spex-8000 mixer mill equipped with a hardened steel vial and two $12.7 \mathrm{~mm}$ diameter balls of $8 \mathrm{~g}$ of mass. In each rub $4 \mathrm{~g}$ of material was sealed under Ar atmosphere and milled for $30 \mathrm{~h}$. The x-ray diffraction characterization was performed with an Ital-Structures $\mathrm{x}$-ray diffractometer using focused and monochromated $\mathrm{Co} \mathrm{K} \alpha_{1}$ radiation and equipped with an INEL CPS120 curved position sensitive detector, which allows the simultaneous detection of the diffracted beam over a range of $120^{\circ}$. The TEM observations were carried out with a JEOL $4000 \mathrm{FX}$ microscope operating at $400 \mathrm{kV}$ and the samples were prepared by dispersing the powder in ethanol and separating ultrasonically the agglomerates. A drop of the suspension was then deposited to dry on a thin carbon film supported by a standard TEM grid. For the SEM observations a Hitachi S-2500 or a Leica 440 scanning electron microscopes were used. The powders were deposited on silver paint on the microscope specimen holder and observed in the secondary electron and CL modes with a beam energy of $20 \mathrm{keV}$ at temperatures between $300 \mathrm{~K}$ and $90 \mathrm{~K}$. The CL measurements were carried out in the visible and near-infrared ranges with a Hamamatsu R928 photomultiplier and a cooled ADC Ge detector, respectively.

\section{Results and discussion}

XRD spectra of both Ge samples, milled wafer and milled powder, show diffraction peaks with the same positions and shapes (figure 1). The difference in the background is explained by the presence of $\mathrm{GeO}_{2}$ in the starting Ge powder which amorphizes during milling. XRD of all milled samples revealed a crystallite average size of about $20 \mathrm{~nm}$. TEM also shows the presence of nanocrystals in the milled samples. Figure 2 shows a high resolution electron microscopy (HREM) image of the milled wafer where nanocrystals of about $10-15 \mathrm{~nm}$ are observed. TEM shows that the average nanocrystal size detected by XRD corresponds to a range of sizes down to few nanometres. The existence of a wide nanocrystal size distribution in ball-milled germanium and silicon has been previously reported [17, 19, 21, 22].

SEM secondary electron images show that the milled powder consists of particles, with a range of sizes up to several microns, formed by aggregation of smaller entities

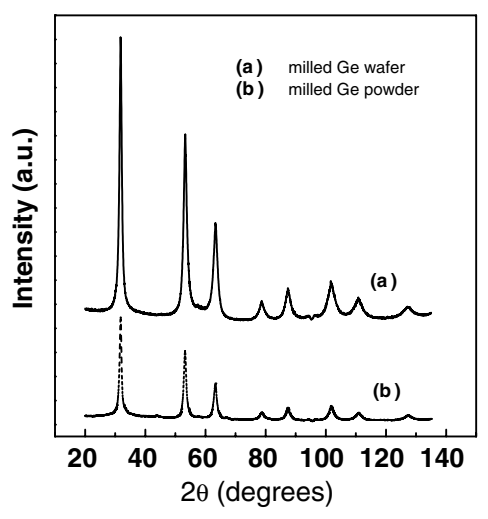

Figure 1. XRD spectra from milled Ge wafer $(a)$ and milled Ge powder $(b)$.

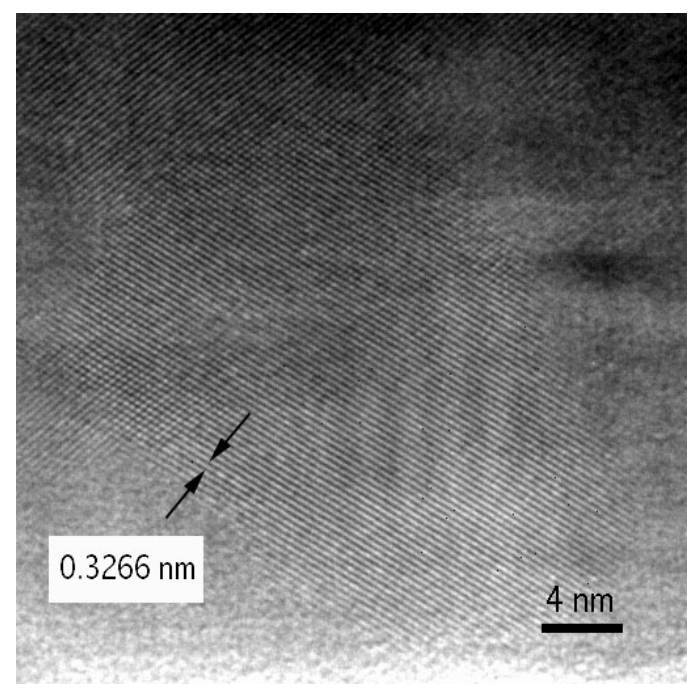

Figure 2. HREM image of milled Ge wafer showing nanoparticles. The interplanar distance is indicated by arrows.

with the typical size of 100 to several hundreds of nanometres (figure 3).

Figure 4 shows the CL spectra of as-received and the milled $\mathrm{GeO}_{2}$ powder. These samples are used as reference to investigate the possible presence of oxide in the milled Ge. Untreated $\mathrm{GeO}_{2}$ shows at $90 \mathrm{~K}$ a single $\mathrm{CL}$ band peaked at $2.43 \mathrm{eV}$ (figure $4(a)$ ). Increasing the temperature causes broadening of the emission band as well as a complex behaviour of the peak, which reaches a lower energy value of $2.35 \mathrm{eV}$ at $230 \mathrm{~K}$ and shifts then towards higher energies. Figure $4(b)$ shows the spectrum at room temperature with an intense band at $2.54 \mathrm{eV}$ and a weak one at $2.99 \mathrm{eV}$ obtained by deconvolution of the broad emission. The spectrum at $90 \mathrm{~K}$ of milled $\mathrm{GeO}_{2}$ (figure $4(a)$ ) shows the main component at $2.49 \mathrm{eV}$ while at room temperature the main emission of the complex band appears at $2.61 \mathrm{eV}$ (figure 4(b)).

As-received Ge powder and wafer did not show any visible luminescence. All milled Ge samples showed luminescence with the main bands in the range 2.4-3.1 eV. Figure 5 shows the CL spectra of samples obtained by milling the wafer. At $90 \mathrm{~K}$ a dominant emission at $2.39 \mathrm{eV}$ is observed and at $295 \mathrm{~K}$ the spectrum consists of an intense band peaked at $3.07 \mathrm{eV}$ and a less intense component at $2.49 \mathrm{eV}$. The spectra of milled 

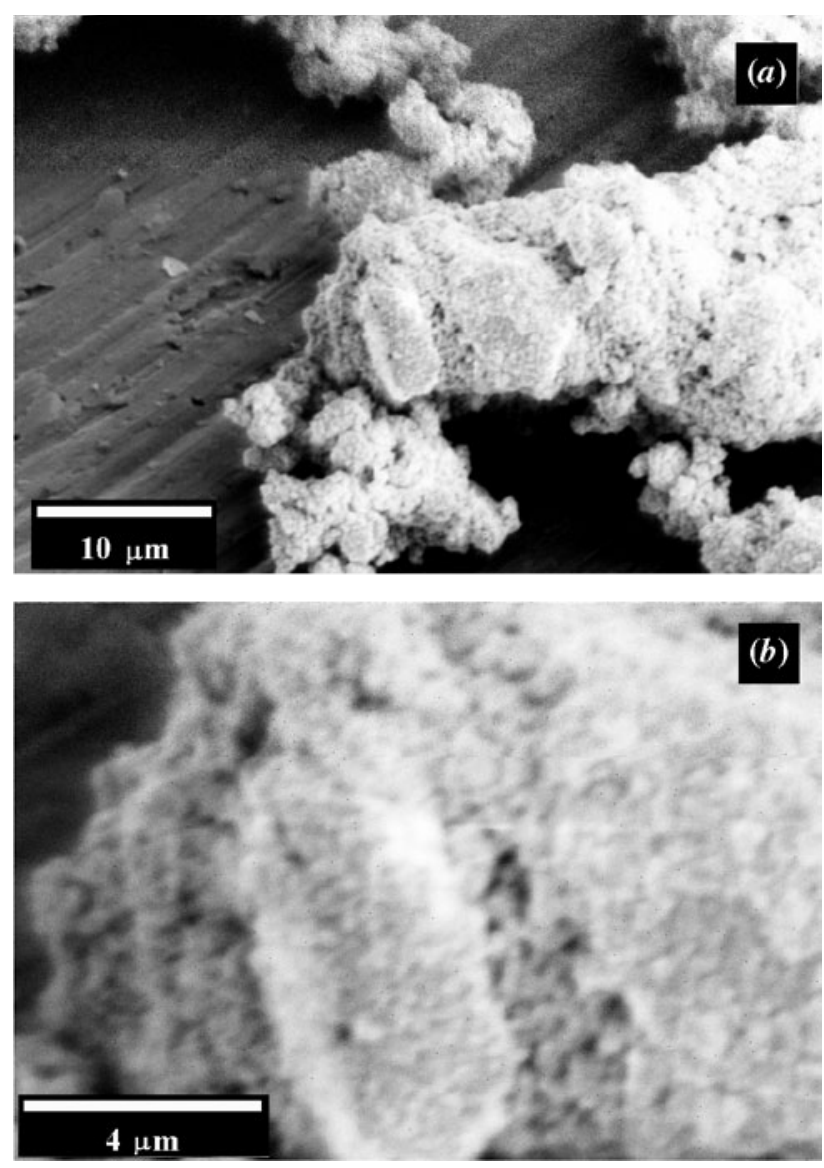

Figure 3. (a) Secondary electron image (SEM) of milled Ge powder. (b) Higher magnification SEM image showing detail of the particles.

Table 1. Summary of the CL peaks reported in this work at 90 and $295 \mathrm{~K}$. The units are $\mathrm{eV}$ and bold numbers indicate dominant transition.

\begin{tabular}{lllll}
\hline & $\mathrm{GeO}_{2}$ & $\begin{array}{l}\text { Milled } \\
\mathrm{GeO}_{2}\end{array}$ & $\begin{array}{l}\text { Milled } \\
\text { Ge wafer }\end{array}$ & $\begin{array}{l}\text { Milled } \\
\text { Ge powder }\end{array}$ \\
\hline $90 \mathrm{~K}$ & $\mathbf{2 . 4 3}$ & 3.02 & 2.86 & 3.14 \\
& - & $\mathbf{2 . 4 9}$ & $\mathbf{2 . 3 9}$ & $\mathbf{2 . 4 7}$ \\
$295 \mathrm{~K}$ & 2.99 & 3.02 & $\mathbf{3 . 0 7}$ & $\mathbf{3 . 1 0}$ \\
& $\mathbf{2 . 5 4}$ & $\mathbf{2 . 6 1}$ & 2.49 & 2.70 \\
\hline
\end{tabular}

Ge powder are shown in figure 6. At $90 \mathrm{~K}$ a high-energy band, which is not resolved in the spectra of the milled wafer, is observed. At room temperature the spectra are similar to those of the milled wafer. Table 1 summarizes the position of the main peaks observed at $90 \mathrm{~K}$ and $295 \mathrm{~K}$ in the investigated $\mathrm{GeO}_{2}$ and $\mathrm{Ge}$ samples. These transitions have been determined from the best fits to experimental CL spectra using a sum of Gaussian line distributions obtained by Jandel Scientific Peakfit program. In fact the spectra show a non-continuous evolution between $90 \mathrm{~K}$ and $295 \mathrm{~K}$, as mentioned above for $\mathrm{GeO}_{2}$ at $230 \mathrm{~K}$. However, the behaviour of different samples can be compared by considering only the luminescence data at the two limit temperatures.

XRD and TEM results show the presence of a nanocrystalline structure in the ball-milled germanium samples, which agrees with the results of Whelan [17]. In
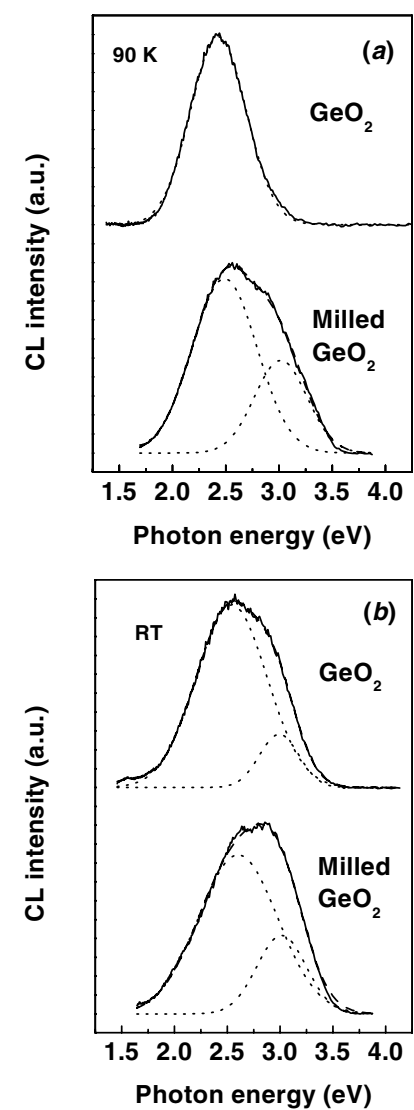

Figure 4. $\mathrm{CL}$ spectra of untreated $\mathrm{GeO}_{2}$ and milled $\mathrm{GeO}_{2}$ at $(a)$ $90 \mathrm{~K}$ and $(b)$ room temperature. Dashed lines show the deconvoluted bands that best fit the experimental results.

[17] a series of milling times up to $200 \mathrm{~h}$ were used, with the aim of obtaining not only nanocrystals but also nanosized particles. However, even after the long milling times a range of particle sizes from tens to hundreds of nanometres was observed by SEM. These particles formed agglomerates with sizes of several microns. In this work a milling time of $30 \mathrm{~h}$ has been found appropriate to produce nanocrystals, which, as shown by TEM and SEM, are part of particles with sizes of the same order as that reported in [17]. Previous work on silicon [21] showed that, under our experimental conditions, the effect of ball milling on nanocrystal and particle size, saturates after milling times of $30 \mathrm{~h}$.

Luminescence from $\mathrm{GeO}_{2}$ has been previously investigated and its relationship with the observed luminescence in Ge nanocrystalline structures has been discussed. Negishi et al [23] studied the emission of oxygen deficient germanium oxide clusters prepared from laser ablation of $\mathrm{GeO}_{2}$, and observed strong photoluminescence at about $2.5 \mathrm{eV}$ which appears as weak emission in the oxidized samples. Fitting et al [24] used CL to investigate $\mathrm{GeO}_{2}$ and found an emission peaked at $2.25 \mathrm{eV}$ in quartzlike crystals that was attributed, according to Skuja model [25], to the presence of oxygen deficient centres. An intense violet luminescence in tetragonal $\mathrm{GeO}_{2}$ crystals was related to twofold-coordinated $\mathrm{Ge}$, possibly generated by oxygen vacancies. They related the same band observed in Ge-doped silica samples to different $\mathrm{Ge}$ phases including $\mathrm{GeO}_{2}$ and Ge nanocrystals. The CL spectrum at $90 \mathrm{~K}$ (figure $4(a)$ ) 

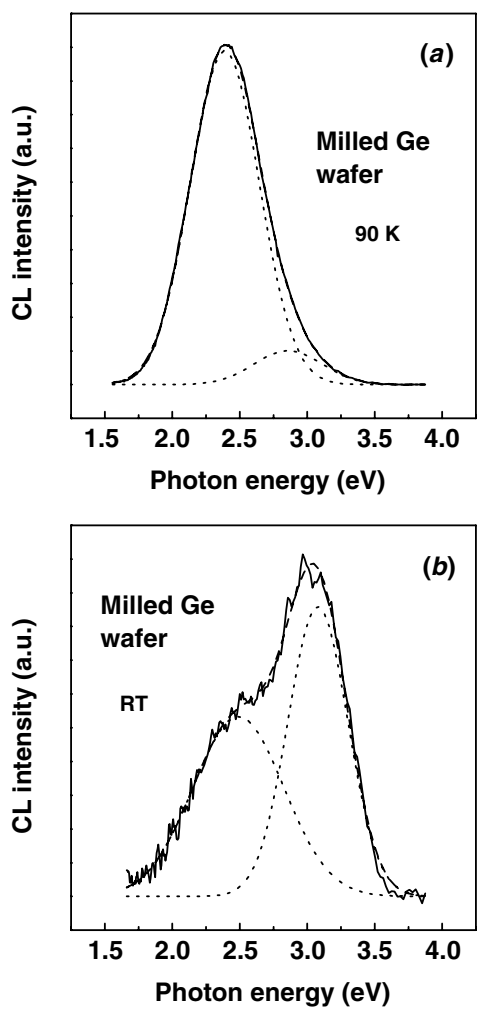

Figure 5. CL spectra of milled Ge wafer at $(a) 90 \mathrm{~K}$ and $(b)$ room temperature. Dashed lines show the deconvoluted bands that best fit the experimental results.

of the untreated $\mathrm{GeO}_{2}$ powder shows a green single band in the energy range reported in the above-mentioned works. Milling causes a spectral broadening that, as deconvolution shows, is mainly due to the growth of a component at about $3 \mathrm{eV}$. This violet component could be related to the partial formation of an amorphous phase during milling. It has been pointed out in [24] that glassy $\mathrm{GeO}_{2}$ resembles in a short-range order sixfold-coordinated germanium in tetragonal structure which shows violet emission. In the spectra of $\mathrm{GeO}_{2}$ at room temperature, the same effect, increase of the $3 \mathrm{eV}$ component after milling, is observed. If milling causes the formation of $\mathrm{GeO}_{2}$ nanocrystals the growth of a $3 \mathrm{eV}$ band agrees with the observation reported in [11] of this band in samples containing $\mathrm{GeO}_{2}$ nanocrystals attributed to a $\mathrm{Ge} / \mathrm{O}$ related defect.

The CL emission of the milled Ge samples is discussed by considering the presence of a nanocrystalline structure and the influence of oxide species at the crystallite surface. As mentioned above, the existence of visible luminescence from Ge nanocrystals has been previously reported. In general the technique used has been PL and the nanocrystals were embedded in a matrix, often of $\mathrm{SiO}_{2}$. In this work we have different experimental conditions: CL in the SEM has been used, which implies a high excitation density, and the nanocrystals were not embedded in a matrix. In fact an objective of this investigation is the study of the properties of Ge nanocrystals obtained by mechanical milling. Kanemitsu et al [7] reported broad PL spectra with a band peaked at 2.2-2.3 eV, related to the presence of Ge nanocrystals in a $\mathrm{SiO}_{2}$ matrix. PL in the range $2.1-2.3 \mathrm{eV}$ in the system $\mathrm{Ge}-\mathrm{SiO}_{2}$ has been observed by other authors [9, 11]. Maeda [8] found
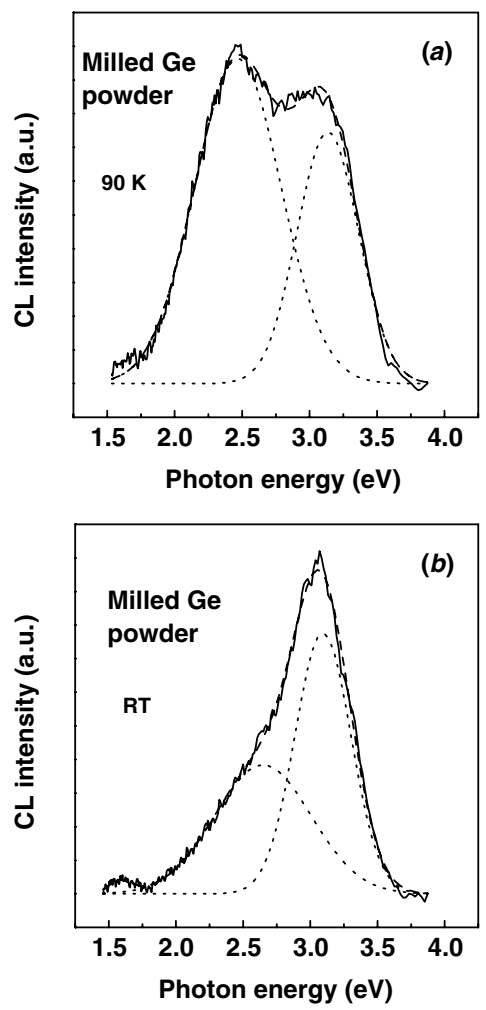

Figure 6. CL spectra of milled Ge powder at (a) $90 \mathrm{~K}$ and (b) room temperature. Dashed lines show the deconvoluted bands that best fit the experimental results.

PL bands between 2.13 and $2.3 \mathrm{eV}$ in Ge nanocrystals in a glassy $\mathrm{SiO}_{2}$ matrix and concluded that the emission properties seemed to be more consistent with a quantum confinement model in Ge than with other models. Discrepancies with a QC model were explained by the presence of non-radiative channels at the nc-Ge surface. A peak at $3.1 \mathrm{eV}$ was observed when a $325 \mathrm{~nm}$ laser was used as excitation source but was not detectable under $488 \mathrm{~nm}$ excitation. A PL band at $3.1 \mathrm{eV}$ was also reported in [11] for nc-Ge in $\mathrm{SiO}_{2}$. The authors proposed a $\mathrm{Ge} / \mathrm{O}$ related defect as the origin of this band. More recently, Choi et al [12] described a $3.0 \mathrm{eV}$ emission from $\mathrm{Ge}$ introduced in $\mathrm{a} \mathrm{SiO}_{2}$ matrix and considered unlikely that germanium oxide is responsible for this band. In [15], colloidal Ge nanocrystals showed luminescence in the $2-2.6 \mathrm{eV}$ range and also at about $3 \mathrm{eV}$. The latter band becomes dominant when high-energy $(350 \mathrm{~nm})$ photons were used for excitation. Oku et al [14] reported 2.6 eV and 3.1 eV PL bands in Ge nanoparticles encapsulated in oxide layers and proposed that the $2.6 \mathrm{eV}$ band originates from the $\mathrm{Ge} / \mathrm{GeO}_{2}$ interface and the $3.1 \mathrm{eV}$ was believed to be due to quantum size effects of the Ge clusters.

As figures 5 and 6 show, milled Ge presents luminescence in the range reported by other authors for Ge nanocrystals in the $\mathrm{SiO}_{2}$ matrix and for nanocrystals encapsulated in oxide layer or in the form of colloidal nanoparticles. On the other hand, the spectra of milled Ge show marked differences with the spectra of untreated and milled $\mathrm{GeO}_{2}$. With the exception of the CL work on oxides, of [24], the above-mentioned results of other authors refer to PL measurements and the comparison with our CL results is not straightforward due to the more selective 
character and the lower excitation energy of PL as compared with CL. We refer first to the emissions about $3.1 \mathrm{eV}$ observed in milled $\mathrm{Ge}$. This band is dominant at room temperature and appears well resolved at $90 \mathrm{~K}$ in the milled powder. In contrast, the blue-violet luminescence in milled or untreated $\mathrm{GeO}_{2}$ is in all cases a relatively weak unresolved component of the spectra. The peak in $\mathrm{GeO}_{2}$ observed by deconvolution appears at about $3 \mathrm{eV}$ while the CL band in milled $\mathrm{Ge}$ is centred at about $3.1 \mathrm{eV}$. These differences indicate that the $3.1 \mathrm{eV}$ band cannot be directly attributed to the presence of $\mathrm{GeO}_{2}$ in milled Ge but the mechanism of emission should involve the presence of Ge nanocrystals. The fact that the $3.1 \mathrm{eV}$ band appears intense or dominates the spectra under the high excitation conditions of the SEM suggests that this emission is similar to the PL band reported in [15], in colloidal nanocrystals, and [8], in nanocrystals in $\mathrm{SiO}_{2}$, which were intense only under excitation with high-energy photons. As summarized above the origin of the PL band at about $3.1 \mathrm{eV}$ observed by other authors is not well determined and quantum size effects in the $\mathrm{Ge}$ clusters as well as $\mathrm{Ge} / \mathrm{O}$ defects have been suggested. Our results show that the $3.1 \mathrm{eV}$ is related to the presence of $\mathrm{Ge}$ nanocrystals but do not enable to confirm that the luminescence is related to a quantum size effect and recombination in the nanocrystal core. Comparison of the $90 \mathrm{~K}$ spectra of both milled Ge samples, milled powder and milled wafer, shows that the $3.1 \mathrm{eV}$ component is more intense in samples prepared from Ge powder. These samples contain more oxygen than those prepared from the wafer which suggests that the presence of oxide in the nanocrystalline structure, in particular states at the nanocrystals/oxide interface, could contribute to the $3.1 \mathrm{eV}$ emissions.

Luminescence emission in the range $2.1-2.5 \mathrm{eV}$ has been observed in this and in previous works to be a property of $\mathrm{Ge}$ nanocrystals and germanium oxide. In the latter case the emission is related to oxygen deficient centres [24]. The green-orange emission has been observed in nanocrystals embedded in oxide matrix and colloidal nanocrystals [15] where the samples are free of oxygen and an oxide layer. Some authors, e.g., [8, 15], attributed emission in this range to the existence of quantum confinement. The presence of oxide on the nanocrystal surface has been also considered to be involved in the emission mechanism [11,14]. Our results show that the nanocrystalline structure produced by mechanical milling leads, as in the case of other systems containing Ge nanocrystals, to luminescence at about $2.4-2.5 \mathrm{eV}$. The CL data do not enable, however, to relate the observed band to a nanocrystal size range or to determine the influence of surface states on the photon energy. As described in the size selected study of [15] a distribution of nanocrystal sizes contributes to a wide luminescence band and in addition it appears that broad PL peaks are characteristic of nanocrystals.

\section{Conclusions}

Nanocrystalline germanium has been produced by mechanical milling of germanium single crystals and germanium powder.
The obtained samples consist of particles with sizes in the micron and submicron range, formed by nanocrystals. The milled samples show two CL bands at about 2.4-2.5 eV and $3.1 \mathrm{eV}$, respectively. Both bands appear related to the presence of nanocrystals. It is suggested that the emission mechanism of the high-energy band involves the presence of oxide at the crystallites surface. Comparison with $\mathrm{CL}$ of $\mathrm{GeO}_{2}$ enables to rule out that the emission of milled Ge is generated in oxide.

\section{Acknowledgment}

This work was supported by MCYT (project MAT2000-2119).

\section{References}

[1] Cullis A G, Canham L T and Calcott P D J 1997 J. Appl. Phys. 82909

[2] Shimizu-Iwayama T, Nakao S and Saitoh K 1994 Appl. Phys. Lett. 651814

[3] Zhao X, Schoenfeld O, Kusano J, Aoyagi Y and Sugano T 1994 Japan. J. Appl. Phys. 33 L649

[4] Cullis A G and Canham L T 1991 Nature 353335

[5] Kanemitsu Y 1994 Phys. Rev. B 4916845

[6] Shimizu-Iwayama T, Kurumado N, Hole D E and Townsend P D 1998 J. Appl. Phys. 836018

[7] Kanemitsu Y, Uto H, Masumoto Y and Maeda Y 1992 Appl. Phys. Lett. 612187

[8] Maeda Y 1995 Phys. Rev. B 511658

[9] Paine D C, Caragianis C, Kim T Y and Shigesato Y 1993 Appl. Phys. Lett. 622842

[10] Yang C M, Shcheglov K V, Brongersma M L, Polman A and Atwater H A 1995 Mat. Res. Soc. Symp. Proc. 358181

[11] Zacharias M and Fauchet P M 1997 Appl. Phys. Lett. 71380

[12] Choi W K, Ho Y W, Ng S P and Ng V 2001 J. Appl. Phys. 89 2168

[13] Lee W S, Yeong J Y, Kim H B, Chae K H, Whang C N, Im S and Song J H 2000 Mater. Sci. Eng. B 69-70 474

[14] Oku T, Nakayama T, Kuno M, Nozue Y, Reine Wallenberg L, Niihara K and Suganuma K 2000 Mater. Sci. Eng. B 74242

[15] Wilcoxon J P, Provencio P P and Samara G A 2001 Phys. Rev. B 64035417

[16] Koch C C 1993 Nanostruct. Mater. 2109

[17] Whelan N J 2000 J. Mater. Res. 152400

[18] Gaffet E and Harmelin M 1990 J. Less-Common Met. 157201

[19] Shen T D, Koch C C, McCormick T L, Nemanich R J, Huang J Y and Huang J G 1995 J. Mater. Res. 10139

[20] Shen T D, Shmagin I, Koch C C, Kolbas R M, Fahmy Y, Bergman L, Nemanich R J, Mc Clure M T, Sitar Z and Quan M X 1977 Phys. Rev. B 557615

[21] Díaz-Guerra C, Montone A, Piqueras J and Cardellini F 2001 Solid State Phenomena 78-79 103

[22] Díaz-Guerra C, Montone A, Piqueras J and Cardellini F Semicond Sci. Technol. 1777

[23] Negishi Y, Nagao S, Nakamura Y, Nakajima A, Kamei S and Kaya K 2000 J. Appl. Phys. 886037

[24] Fitting H J, Barfels T, Trukhin A N and Schmidt B 2001 J. Non-Cryst. Solids 27951

[25] Skuja L 1994 J. Non-Cryst. Solids 167229 